\title{
QUASI-CONFORMAL DEFORMATIONS OF NONLINEARIZABLE GERMS
}

\author{
KINGSHOOK BISWAS
}

(Communicated by Mario Bonk)

\begin{abstract}
Let $f(z)=e^{2 \pi i \alpha} z+O\left(z^{2}\right), \alpha \in \mathbb{R}$, be a germ of a holomorphic diffeomorphism in $\mathbb{C}$. For $\alpha$ rational and $f$ of infinite order, the space of conformal conjugacy classes of germs topologically conjugate to $f$ is parametrized by the Ecalle-Voronin invariants (and in particular is infinite-dimensional). When $\alpha$ is irrational and $f$ is nonlinearizable it is not known whether $f$ admits quasiconformal deformations. We show that if $f$ has a sequence of repelling periodic orbits converging to the fixed point, then $f$ embeds into an infinite-dimensional family of quasi-conformally conjugate germs, no two of which are conformally conjugate.
\end{abstract}

\section{INTRODUCTION}

Let $f(z)=e^{2 \pi i \alpha} z+O\left(z^{2}\right), \alpha \in \mathbb{R} / \mathbb{Z}$, be a germ of a holomorphic diffeomorphism fixing the origin in $\mathbb{C}$. We consider the question of when $f$ admits quasi-conformal deformations; i.e. when do there exist germs $g$ which are quasi-conformally but not conformally conjugate to $f$ ? If $f$ is linearizable (i.e. analytically conjugate to the rigid rotation $R_{\alpha}(z)=e^{2 \pi i \alpha} z$ ), then any germ topologically conjugate to $f$ is linearizable, in particular conformally conjugate to $f$, so $f$ is rigid and does not admit nontrivial deformations. In the nondegenerate parabolic case (i.e. $\left.\alpha=p / q \in \mathbb{Q}, f^{q} \neq i d\right)$, the quasi-conformal conjugacy class of $f$ contains an infinite-dimensional family of conformal conjugacy classes parametrized by the Ecalle-Voronin invariants ([2], 6]). In the irrationally indifferent nonlinearizable case ( $\alpha$ irrational, $f$ not linearizable), it seems to be unknown whether quasiconformal deformations are possible. We show the following:

Theorem 1.1. Let $f$ be an irrationally indifferent nonlinearizable germ with a sequence of repelling periodic orbits accumulating the origin. Then there is a family of quasi-conformal maps $\left\{H_{\Xi}\right\}_{\Xi \in \mathcal{D}}$ parametrized by an infinite polydisc $\mathcal{D}=\{\Xi=$ $\left.\left(\xi_{n}\right)_{n \geq 1}: \xi_{n} \in \mathbb{C},\left|\xi_{n}\right|<1\right\}$ such that all conjugates $G_{\Xi}=H_{\Xi} \circ f \circ H_{\Xi}^{-1}$ are holomorphic, and two such germs $G_{\Xi_{1}}, G_{\Xi_{2}}$ are conformally conjugate if and only if $\Xi_{1}=\Xi_{2}$. For fixed $z, H_{\Xi}(z)$ depends holomorphically on each $\xi_{n}$.

A very rough outline of the proof is as follows: given $\Xi=\left(\xi_{n}\right)$ the germ $G_{\Xi}$ is obtained by quasi-conformally deforming $f$ near its repelling periodic orbits. Each such periodic orbit is attracting for $f^{-1}$, and it is possible to construct an $\mathrm{R}$
2012.

Received by the editors September 13, 2011 and, in revised form, April 18, 2012 and May 27,

2010 Mathematics Subject Classification. Primary 37F50.

This research was partly supported by the Department of Science and Technology research project grant DyNo. 100/IFD/8347/2008-2009. 
$f$-invariant Beltrami differential on each basin of attraction such that the quasiconformal map $H_{\Xi}$ rectifying the Beltrami differential conjugates $f$ to a holomorphic germ $G_{\Xi}$ having multipliers at the periodic orbits, each depending on only one $\xi_{n}$, with the dependence univalent. The multipliers at periodic orbits being invariant under conformal conjugacies, the conclusion of the theorem follows.

Examples of germs satisfying the hypothesis of the theorem are germs of rational maps of degree $d$ with $\alpha$ satisfying the Cremer condition of degree $d$ (see for example Milnor [3, Ch. 8),

$$
\limsup \frac{\log \log q_{n+1}}{q_{n}}>\log d
$$

(where $\left(p_{n} / q_{n}\right)$ are the continued fraction convergents of $\alpha$ ), which ensures that the fixed point is accumulated by periodic orbits (only finitely many of which can be nonrepelling for a rational map).

Perez-Marco has shown ([5]) for any nonlinearizable germ $f$ the existence of a unique monotone one-parameter family $\left(K_{t}\right)_{t>0}$ of full, totally invariant continua called hedgehogs containing the fixed point. In [1] it is proved that any conformal mapping in a neighbourhood of a hedgehog $K$ of a germ $f_{1}$ mapping $K$ to a hedgehog of a germ $f_{2}$ necessarily conjugates $f_{1}$ to $f_{2}$. As a corollary of Theorem 1.1 we have

Theorem 1.2. There exists a holomorphic motion $\phi: \mathbb{D} \times \hat{\mathbb{C}} \rightarrow \hat{\mathbb{C}}$ of $\hat{\mathbb{C}}$ over $\mathbb{D}$ and a hedgehog $K$ such that all the sets $\phi(t, K)$ are hedgehogs, all of which are quasi-conformal images of $K$, but for $s \neq t, \phi(s, K)$ cannot be conformally mapped to $\phi(t, K)$.

\section{Deformations}

We fix a germ $f(z)=e^{2 \pi i \alpha} z+O\left(z^{2}\right), \alpha \in \mathbb{R}-\mathbb{Q}$, and a neighbourhood $U$ of the origin such that $f$ and $f^{-1}$ are univalent on a neighbourhood $V$ of $\bar{U}$. By a periodic orbit or cycle of $f$ of order $q \geq 1$ we mean a finite set $\mathcal{O}=\left\{z_{1}, \ldots, z_{q}\right\} \subset U$ such that $f\left(z_{i}\right)=z_{i+1}, 1 \leq i \leq q-1$, and $f\left(z_{q}\right)=z_{1}$. The multiplier at the periodic orbit is defined to be $\lambda=f^{\prime}\left(z_{1}\right) f^{\prime}\left(z_{2}\right) \ldots f^{\prime}\left(z_{q}\right)=\left(f^{q}\right)^{\prime}\left(z_{i}\right)$. The periodic orbit is called attracting, indifferent or repelling according as $|\lambda|<1,|\lambda|=1$ or $|\lambda|>1$ respectively. A periodic orbit for $f$ of multiplier $\lambda$ is a periodic orbit for $f^{-1}$ of multiplier $\lambda^{-1}$. The basin of attraction of an attracting periodic cycle is defined by $\mathcal{A}(\mathcal{O}, f):=\left\{z \in U: f^{n}(z) \rightarrow \mathcal{O}\right.$ as $\left.n \rightarrow+\infty\right\}$.

Lemma 2.1. Given $q \geq 1$, the set of multipliers of cycles of order $q$ of $f$ in $U$ is finite.

Proof. Suppose not, and let $\mathcal{O}_{n}$ be a sequence of cycles of $f$ of order $q$ such that the multipliers $\lambda_{n}$ of $\mathcal{O}_{n}$ are all distinct. Choose $z_{n} \in \mathcal{O}_{n}$; then passing to a subsequence we may assume $z_{n} \rightarrow \xi \in \bar{U}$. Since $f\left(z_{n}\right) \in U$ for all $n$, it follows that $f(\xi) \in \bar{U}$; similarly it follows that $f$ can be iterated $q$ times near $\xi$. Let $f^{q}$ be the germ defined near $\xi$ given by iterating $q$ times $f$ near $\xi$; then $f^{q}$ has a sequence of fixed points $z_{n}$ accumulating $\xi$. Hence $f^{q} \equiv i d$, so $\left(f^{q}\right)^{\prime} \equiv 1$ and $\lambda_{n}=1$ for all $n$ large, a contradiction.

Since $f$ is asymptotic to an irrational rotation, i.e. $f(z) / z \rightarrow e^{2 \pi i \alpha}$ as $z \rightarrow 0$, it follows that if $f$ has small cycles, then the orders of the cycles must go to infinity. 
2.1. Deforming repelling periodic orbits. Let $\mathbb{H}$ denote the upper half-plane and let $E: \mathbb{H} \rightarrow\{|z|>1\}$ denote the universal covering defined by $E(z)=e^{-2 \pi i z}$. For $r>0$ and $\tau \in \mathbb{H}$, let $B(\tau, r)$ denote the ball of radius $r$ around $\tau$ with respect to the hyperbolic metric on $\mathbb{H}$. Given a repelling periodic orbit $\mathcal{O}$ of $f$ with multiplier $\lambda$, choose $\tau \in \mathbb{H}$ such that $E(\tau)=\lambda$ and $0<r<1$ such that $E$ is injective on $B(\tau, r)$. For $\tau^{\prime} \in B(\tau, r)$, we deform the multiplier of $f$ quasi-conformally from $\lambda$ to $\lambda^{\prime}=E\left(\tau^{\prime}\right)$ by constructing a corresponding $f$-invariant Beltrami differential $\mu=\mu\left(\mathcal{O}, \tau, \tau^{\prime}\right)$ on the basin of attraction $\mathcal{A}\left(\mathcal{O}, f^{-1}\right)$ as follows:

By Köenigs linearization theorem (see [3], Ch. 6), for any repelling periodic orbit $\mathcal{O}$ of $f$ with multiplier $\lambda$ there exists a unique holomorphic map $\phi$ defined on a neighbourhood of $\mathcal{O}$ such that $\phi\left(z_{i}\right)=0, \phi^{\prime}\left(z_{i}\right)=1, i=1, \ldots, n$, and $\phi\left(f^{q}(z)\right)=$ $\lambda \phi(z)$. The conformal isomorphism $L: \mathbb{C}^{*} \rightarrow \mathbb{C} / \mathbb{Z}, w \mapsto \xi=-\frac{1}{2 \pi i} \log w$ conjugates the linear map $w \mapsto \lambda w$ on $\mathbb{C}^{*}$ to the translation $\xi \mapsto \xi+\tau$ on $\mathbb{C} / \mathbb{Z}$.

Let $\tau^{\prime} \in B(\tau, r)$ and let $K$ be the real linear map on $\mathbb{C}$ defined by $K(1)=$ $1, K(\tau)=\tau^{\prime}$. Then $K$ commutes with the translation by one and hence gives a quasi-conformal orientation preserving (since $\Im \tau, \Im \tau^{\prime}>0$ ) homeomorphism $\tilde{K}$ : $\mathbb{C} / \mathbb{Z} \rightarrow \mathbb{C} / \mathbb{Z}$. The Beltrami differential of $\tilde{K}$ is constant and invariant under translations of $\mathbb{C} / \mathbb{Z}$, and $\tilde{K}$ conjugates the translation $\xi \mapsto \xi+\tau$ on $\mathbb{C} / \mathbb{Z}$ to $\xi \mapsto \xi+\tau^{\prime}$.

We let $\mu$ be the Beltrami differential of $\tilde{K} \circ L \circ \phi$ restricted to a small neighbourhood $D$ of $z_{1} \in \mathcal{O}$. The map $\tilde{K} \circ \phi$ conjugates $f^{q}$ to the translation $\xi \mapsto \xi+\tau^{\prime}$. So at points $z, z^{\prime}=f^{q}(z)$ in $D, \mu$ satisfies the invariance condition

$$
\mu\left(z^{\prime}\right) \overline{\left(f^{q}\right)^{\prime}(z)}=\mu(z)\left(f^{q}\right)^{\prime}(z) .
$$

So $\mu$ extended to the neighbourhood $V=D \cup f(D) \cup \cdots \cup f^{q-1}(D)$ of $\mathcal{O}$ by putting

$$
\mu\left(f^{j}(z)\right)=\mu(z) \frac{\left(f^{j}\right)^{\prime}(z)}{\left(f^{j}\right)^{\prime}(z)}
$$

for $z \in D, j=1, \ldots, q-1$ is an $f$-invariant Beltrami differential. Similarly the above equation allows us to extend $\mu$ to an $f$-invariant Beltrami differential $\mu\left(\mathcal{O}, \tau, \tau^{\prime}\right)$ on $\mathcal{A}\left(\mathcal{O}, f^{-1}\right)=\bigcup_{n>1} f^{n}(V)$. Note that since the hyperbolic distance between $\tau$ and $\tau^{\prime}$ is bounded by one, $\mu$ has $L^{\infty}$ norm less than $\kappa$ for some fixed constant $0<\kappa<1$.

Lemma 2.2. Any quasi-conformal homeomorphism $h$ with a Beltrami coefficient equal to $\mu=\mu\left(\mathcal{O}, \tau, \tau^{\prime}\right)$ on a neighbourhood of $\mathcal{O}$ conjugates $f$ to a map $g=h \circ f \circ h^{-1}$ with a periodic orbit $h(\mathcal{O})$ of multiplier $\lambda^{\prime}=E\left(\tau^{\prime}\right)$. The dependence of $\mu\left(\mathcal{O}, \tau, \tau^{\prime}\right)$ on $\tau^{\prime}$ is holomorphic.

Proof. For $i=1, \ldots, q$ we let $\psi_{i}$ be the branch of $\phi^{-1}$ sending 0 to $z_{i}$. By construction the map $k$ defined by $k=\psi_{i} \circ L^{-1} \circ \tilde{K} \circ L \circ \phi(z)$ for $z$ in a neighbourhood of $z_{i}$ has a Beltrami coefficient equal to $\mu$ and conjugates $f$ on a neighbourhood of $\mathcal{O}$ to a holomorphic map $f_{1}=k \circ f \circ k^{-1}$ with periodic orbit $k(\mathcal{O})$ and multiplier $\lambda^{\prime}=E\left(\tau^{\prime}\right)$. Since $h$ and $k$ have the same Beltrami coefficient, the map $h \circ k^{-1}$ is holomorphic and conjugates $f_{1}$ to $g$, so the multipliers of $f_{1}$ and $g$ are equal. The Beltrami differential of $\tilde{K}$ is constant equal to $\frac{\tau-\tau^{\prime}}{2 \Re \tau+\left(\tau^{\prime}-\tau\right)}$, which depends holomorphically on $\tau^{\prime}$, so the Beltrami differential $\mu\left(\mathcal{O}, \tau, \tau^{\prime}\right)$ of $\tilde{K} \circ L \circ \phi$ depends holomorphically on $\tau^{\prime}$. 
2.2. Deforming germs with small cycles. We use the Beltrami differentials $\mu\left(\mathcal{O}, \tau, \tau^{\prime}\right)$ to deform a germ with small cycles as follows:

Let $f$ be an irrationally indifferent germ with a sequence of repelling small cycles of orders $\left(q_{n}\right)$ (which we may assume to be strictly increasing). Let $U$ be a neighbourhood of the origin such that $f$ and $f^{-1}$ are univalent on a neighbourhood of $\bar{U}$. Let $L_{n} \subset\{|z|>1\}$ be the finite set of multipliers of all repelling period $q_{n}$ orbits of $f$ in $U$. Choose a finite set $T_{n} \subset \mathbb{H}$ and $0<r_{n}<1$ such that $E$ maps $T_{n}$ bijectively onto $L_{n}$, the balls $B\left(\tau, r_{n}\right), \tau \in T_{n}$, are disjoint, and $E$ is injective on the union of these balls.

Let $I=\left\{\iota=(n, \tau): n \geq 1, \tau \in T_{n}\right\}$, and for each $\iota=(n, \tau) \in I$, choose a conformal map $S_{\iota}$ of the unit disk $\mathbb{D}$ onto $B\left(\tau, r_{n}\right)$ such that $S_{\iota}(0)=\tau$. Let $\mathcal{D}_{1}$ be the infinite polydisk $\mathcal{D}_{1}=\left\{\Omega=\left(\omega_{\iota}\right)_{\iota \in I}: \omega_{\iota} \in \mathbb{D}\right\}$. The basins of attraction $\mathcal{A}\left(\mathcal{O}, f^{-1}\right)$ of distinct repelling cycles of $f$ are disjoint, so for any $\Omega=\left(\omega_{\iota}\right)_{\iota \in I} \in \mathcal{D}_{1}$ we can define an $f$-invariant Beltrami differential $\mu_{\Omega}$ on $U$ as follows:

We put, for $z$ belonging to $\mathcal{A}\left(\mathcal{O}, f^{-1}\right)$, where $\mathcal{O}$ is a repelling cycle for $f$ of order $q_{n}$ and multiplier $E(\tau)$ where $\tau \in T_{n}$,

$$
\mu_{\Omega}(z):=\mu\left(\mathcal{O}, \tau, S_{\iota}\left(\omega_{\iota}\right)\right)(z),
$$

where $\iota=(n, \tau)$, and put $\mu_{\Omega}(z)=0$ otherwise.

Note that since the hyperbolic distance between $\tau$ and $S_{\iota}\left(\omega_{\iota}\right)$ is bounded by 1, $\mu_{\Omega}$ has $L^{\infty}$ norm less than $\kappa$ for a fixed constant $0<\kappa<1$. Let $h_{\Omega}$ be the unique quasi-conformal homeomorphism with Beltrami coefficient $\mu_{\Omega}$ fixing $0,1, \infty$ given by the Measurable Riemann Mapping Theorem. Then $g_{\Omega}=h_{\Omega} \circ f \circ h_{\Omega}^{-1}$ is a holomorphic germ fixing the origin with small cycles. By Naishul's theorem [4] the multiplier at an indifferent fixed point is a topological conjugacy invariant, so $g_{\Omega}^{\prime}(0)=e^{2 \pi i \alpha}$. Note that if $\mathcal{O}$ is any repelling cycle for $g_{\Omega}$ of order $q_{n}$ contained in $h_{\Omega}(U)$, then the multiplier of $g_{\Omega}$ at $\mathcal{O}$ is $E\left(S_{\iota}\left(\omega_{\iota}\right)\right)$ for some $\iota=(n, \tau) \in I$.

Lemma 2.3. For $\Omega_{1}=\left(\omega_{\iota, 1}\right)_{\iota \in I}, \Omega_{2}=\left(\omega_{\iota, 2}\right)_{\iota \in I} \in \mathcal{D}_{1}$, if the germs $g_{1}=g_{\Omega_{1}}, g_{2}=$ $g_{\Omega_{2}}$ are holomorphically conjugate, then for all $n$ large there exists $\iota=\left(n, \tau_{n}\right) \in I$ such that $\omega_{\iota, 1}=\omega_{\iota, 2}$.

Proof. Let $\phi$ be a holomorphic germ conjugating $g_{1}$ to $g_{2}$. Let $h_{1}=h_{\Omega_{1}}, h_{2}=h_{\Omega_{2}}$. Choose a small neighbourhood $W \subset U$ of the origin such that $\phi$ is univalent on $h_{1}(W)$ and $\phi\left(h_{1}(W)\right) \subset h_{2}(U)$. For all $n$ large, $f$ has a repelling cycle $\mathcal{O}_{n}$ of order $q_{n}$ contained in $W$, with multiplier $\lambda_{n}=E\left(\tau_{n}\right)$ for some $\tau_{n} \in T_{n}$. For such an $n, h_{1}\left(\mathcal{O}_{n}\right)$ is a repelling cycle of $g_{1}$ of order $q_{n}$ with multiplier $E\left(S_{\iota}\left(\omega_{\iota, 1}\right)\right)$, where $\iota=\left(n, \tau_{n}\right) \in I$. Now $\phi\left(h_{1}\left(\mathcal{O}_{n}\right)\right)$ is a repelling cycle of $g_{2}$ of order $q_{n}$ contained in $h_{2}(U)$, and hence must have multiplier $E\left(S_{\iota^{\prime}}\left(\omega_{\iota^{\prime}, 2}\right)\right)$ for some $\iota^{\prime}=\left(n, \tau_{n}^{\prime}\right) \in I$, so it follows that $E\left(S_{\iota}\left(\omega_{\iota, 1}\right)\right)=E\left(S_{\iota^{\prime}}\left(\omega_{\iota^{\prime}, 2}\right)\right)$. Since $E$ is injective on the union of the balls $B\left(\tau, r_{n}\right), \tau \in T_{n}$, we must have $\left.S_{\iota}\left(\omega_{\iota, 1}\right)\right)=S_{\iota^{\prime}}\left(\omega_{\iota^{\prime}, 2}\right)$, and since these balls are disjoint it follows that $\tau_{n}^{\prime}=\tau_{n}, \iota^{\prime}=\iota, \omega_{\iota, 1}=\omega_{\iota, 2}$.

We can now prove Theorem 1.1

Proof. Let $\mathcal{D}$ be the infinite polydisc $\mathcal{D}=\left\{\Xi=\left(\xi_{m}\right)_{m \geq 1}: \xi_{m} \in \mathbb{D}\right\}$. Let $\left(p_{m}\right)_{m \geq 1}$ be an infinite sequence of distinct primes, and define a map $R: \mathcal{D} \rightarrow \mathcal{D}_{1}$ as follows: $R(\Xi):=\Omega$, where $\omega_{\iota}=\xi_{m}$ if $\iota=(n, \tau)$ where $n$ is a power of $p_{m}$, and $\omega_{\iota}=0$ otherwise. Define $H_{\Xi}:=h_{R(\Xi)}$. Then the conjugates $G_{\Xi}=H_{\Xi} \circ f \circ H_{\Xi}^{-1}=g_{R(\Xi)}$ are holomorphic. 
Suppose for $\Xi_{1}, \Xi_{2} \in \mathcal{D}$, the germs $G_{\Xi_{1}}, G_{\Xi_{2}}$ are holomorphically conjugate. Then $\Omega_{1}=R\left(\Xi_{1}\right), \Omega_{2}=R\left(\Xi_{2}\right)$ satisfy the conclusion of Lemma 2.3 above, and it follows easily from the definition of $R$ that $\Xi_{1}=\Xi_{2}$.

It follows from Lemma 2.2 that $\mu_{\Omega}$ restricted to any basin $A=\mathcal{A}\left(\mathcal{O}, f^{-1}\right)$ of a repelling cycle $\mathcal{O}$ for $f$ of order some $q_{n}$ depends holomorphically (as an element of $\left.L^{\infty}(A)\right)$ on each $\omega_{\iota}$ for $\iota$ of the form $(n, \tau)$. It then follows easily from the definition of the map $R$ that $\mu_{R(\Xi)}$ depends holomorphically (as an element of $L^{\infty}(\hat{\mathbb{C}})$ ) on each $\xi_{m}$, and hence by the Measurable Riemann Mapping Theorem so does $H_{\Xi}(z)$ for fixed $z$.

We now prove Theorem 1.2

Proof. Let $f$ be as above and $K \subset U$ be a hedgehog of $f$. For $t \in \mathbb{D}$ we let $\Xi_{t}$ be the constant sequence $\left(\xi_{m}=t\right)$. Then it is easy to see (by considering the restriction to basins of attraction) that $\mu_{R\left(\Xi_{t}\right)}$ depends holomorphically on $t$, and $\phi:(t, z) \mapsto H_{\Xi_{t}}(z)$ gives the required holomorphic motion.

\section{ACKNOWLEDGEMENTS}

The author thanks Ricardo Perez-Marco for many helpful discussions and comments. This article was written in part during a visit to the Chennai Mathematical Institute. The author is very grateful to the organizers for their warmth and hospitality.

\section{REFERENCES}

[1] Kingshook Biswas, Complete conjugacy invariants of nonlinearizable holomorphic dynamics, Discrete Contin. Dyn. Syst. 26 (2010), no. 3, 847-856, DOI 10.3934/dcds.2010.26.847. MR2600719 (2011c:37091)

[2] J. Écalle, Théorie itérative: introduction à la théorie des invariants holomorphes (French), J. Math. Pures Appl. (9) 54 (1975), 183-258. MR0499882 (58 \#17637)

[3] John Milnor, Dynamics in one complex variable, Introductory lectures, Friedr. Vieweg \& Sohn, Braunschweig, 1999. MR:1721240 (2002i:37057)

[4] V. I. Naishul, Topological invariants of analytic and area-preserving mappings and their application to analytic differential equations in $\mathbb{C}^{2}$ and $\mathbb{C P}^{2}$, Trans. Moscow Math. Soc. 42 (1983), 239-250. MR0656288 (84f:58092)

[5] Ricardo Pérez-Marco, Fixed points and circle maps, Acta Math. 179 (1997), no. 2, 243-294, DOI 10.1007/BF02392745. MR1607557 (99a:58130)

[6] S. M. Voronin, Analytic classification of germs of conformal mappings $(\mathbf{C}, 0) \rightarrow(\mathbf{C}, 0)$ (Russian), Funktsional. Anal. i Prilozhen. 15 (1981), no. 1, 1-17, 96. MR609790 (82h:58008)

Department of Mathematics, Ramakrishna Mission Vivekananda University, Belur Math, Howrah 711202, India

E-mail address: kingshook@rkmvu.ac.in 